\title{
Employing Domain Specific Discriminative Information to Address Inherent Limitations of the LBP Descriptor in Face Recognition
}

\author{
Junjie Fan and Ognjen Arandjelović ${ }^{\dagger}$ \\ School of Computer Science \\ University of St Andrews \\ North Haugh \\ St Andrews KY16 9SX \\ Fife, Scotland \\ United Kingdom \\ †ognjen.arandjelovic@gmail.com
}

\begin{abstract}
The local binary descriptor (LPB) and its derivatives have a demonstrated track record of good performance in face recognition. Nevertheless the original descriptor, the framework within which it is employed, and the aforementioned improvements of these in the existing literature, all suffer from a number of inherent limitations. In this work we highlight these and propose novel ways of addressing them in a principled fashion. Specifically, we introduce (i) gradient based weighting of local descriptor contributions to region based histograms as a means of avoiding data smoothing by non-discriminative image loci, and (ii) Gaussian fuzzy region membership as a means of achieving robustness to registration errors. Importantly, the nature of these contributions allows the proposed techniques to be combined with the existing extensions to the LBP descriptor thus making them universally recommendable. Effectiveness is demonstrated on the notoriously challenging Extended Yale B face corpus.
\end{abstract}

\section{INTRODUCTION}

Face recognition is often described as one of the most active areas of research in computer vision [1], [2], [3], [4]. While we are unaware of attempts to formalize this claim and support it with rigorous empirical evidence, it is beyond doubt that the field has undergone substantial changes over time. By this we are not referring merely to changes in the technical approach which can be naturally expected to take place as advances are made, but rather to the practical paradigms and the context in which face recognition is employed.

Early face recognition work can be described as a proverbial exploratory mission which served to deepen the understanding of the key challenges and features (in an abstract sense) which have the greatest discriminative power [5], [6]. Geometric features and the first statistical appearance based methods were described in this period. Thereafter the focus has shifted to the practical challenge of making face recognition useful in real world security oriented applications. It is in this period that the difficulty of the problem has crystallized, with concurrent changes in pose, illumination, resolution, and other extrinsic factors, exposing the limitations of the proposed algorithms [7], [8], [9], [10]. Most face recognition work falls under the umbrella of this conceptual period. Despite the immense amount of research effort, both by academia and industry, the highly optimistic predictions expressed in the early years of face recognition research failed to materialize: in unconstrained conditions the performance of face recognition in security applications remains disappointing [11], [12]. The key reason lies in the nature of the demands of most security applications on the one hand, and the inherent discriminative weakness of facial biometrics. As regards the former, security applications demand a low false positive rate (allowing an intruder the access to a resource carries a high cost) and often a low false negative rate (denying access to a legitimate user is frustrating, time consuming, and potentially costly) [13]. At the same time, on the latter point, there is no compelling evidence that face based biometrics even in principle can be used to attain these demands. Face recognition by humans, often intuitively seen as highly sophisticated, is in fact not very accurate when evaluated in conditions comparable to those in which automatic methods are expected to operate [14], [15]. Humans use a variety of constraints, such as knowledge based priors ("whom do I expect to encounter in this place?'), complementary biometrics (height, gait, voice, etc.), and a plethora of others to simplify the task in everyday situations. However, such assumptions are either difficult to incorporate in automatic methods (e.g. due to the semantic gap) or inappropriate in the context of practical applications of interest. 
A turning point for face recognition research has come in the last decade with the emergence of massive amounts of visual data - the focus has shifted to the use of face recognition for the retrieval and organization of photographs and video recordings [16], [4], [17]. The requirements of these applications contrast the aforementioned requirements of security applications: following the successes of web search engines, by adopting the ranked retrieval presentation of output, both socalled type I and type II errors are much more readily tolerated [13]. The user is often not overly troubled by not every instance of interest being retrieved, or it not being retrieved at rank-1, as long as correct matches are within a reasonable rank (the quantified meaning of 'reasonable' being somewhat dependent on the application). Thus, to summarise briefly the history of face recognition, the field has largely been characterized by incremental (but important and cumulatively significant) technical advances with major practical leaps which came though by innovative ways of seeing the same problem though a different lens.

Notwithstanding the contextual paradigm sifts just described, work on the underlying fundamentals of face recognition continues with unabated effort [18], [19], [20], [21], [22], with many outstanding challenges. In recent years, particularly promising innovations are arising from the use of sparse coding [2], [21], [23], dictionary representations [24], [25], and deep learning [26], [27], [28], [17]. Nevertheless many of these approaches suffer from a variety of practical limitations, including their limited interpretability and often seemingly inexplicable failure modes []. Consequently, conceptually simpler and often computationally more efficient approaches remain attractive, especially when enveloped within frameworks which clearly make use of contextual information [29]. In this paper we focus on one of such approaches, or rather umbrella of approaches underlain by a well-known local descriptors known as local binary patterns (LBP). Building upon previous work and the successes of LBP based methods, we start by highlighting a number of inherent limitations of this group of methods, and hence propose a series of improvements which address these in a simple, principled, and effective manner.

\section{BACKGROUND}

It is worth beginning by noting that the term local binary pattern is frequently employed somewhat loosely, perhaps particularly so within the context of face recognition, in that its strict and original reference is that to a type of local descriptor, whereas in some instances it is used to refer to the overarching framework which is underlain by the aforementioned descriptor but seeks to describe holistic image appearance ${ }^{1}$.

The LBP descriptor was originally proposed in the context of texture recognition but has since been shown as very

\footnotetext{
${ }^{1}$ Compare this with the even more widely known SIFT, which refers to descriptor of local appearance (or rather the transformation of local appearance into a low-dimensional descriptor), and the popular object or scene recognition algorithm introduced by Lowe [30].
}
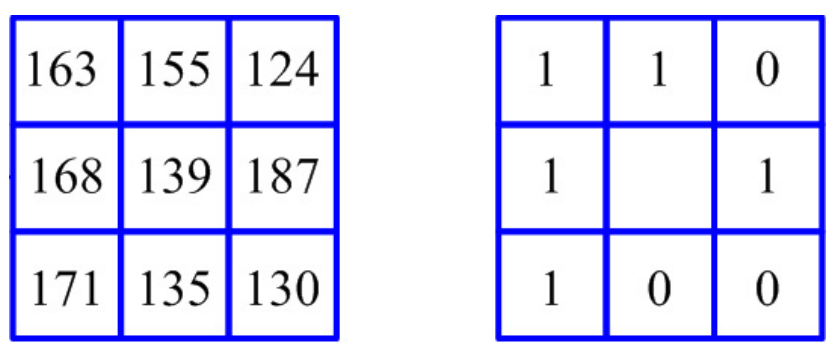

Fig. 1. Example of a $3 \times 3$ image patch (appearance) and the corresponding local binary pattern. The neighbourhood is binarized by comparing the value a pixel and the values of each of its 8 neighbours.

effective across a range of applications, including face recognition [31], [32], object recognition, and numerous others. The original descriptor considers image patches of size $3 \times 3$ pixels. By comparing the values of the 8 neighbouring pixels with the value of the central pixel, the neighbourhood is mapped to a series of binary digits ( 0 or 1$)$ depending on whether a specific pixel has a smaller value than the central pixel or not, as illustrated in Figure 1. The 8 bit sequence corresponds to an integer in the range $[0,127]$ and can be used to describe the local appearance.

The description of an entire image (or a region of interest within it) is then obtained by creating histograms over local LBP descriptors within blocks into which the image is divided, with the number of blocks being a free parameter, learnt from training data or set a priori.

\section{A. Improvements and derivative methods}

Owing to the general effectiveness of LBPs, a number of derivative methods have been proposed [33]. Many of these focused on low-level tweaks of the descriptor itself and how specifically binarization of a pixel neighbourhood is performed. For example, modified local binary patterns $(\mathrm{mLBP})$ use the mean $3 \times 3$ pixel neighbourhood average as the reference comparison value, rather than its central pixel [34], whereas transition local binary patterns (tLBP) perform binarization not by means of comparisons with the central pixel but rather on clockwise comparisons between neighbouring pixels themselves [35].

Another prominent direction of improvements addresses the thresholding of pixels which lies at the heart of LBPs and makes them vulnerable to small perturbations when compared pixels have values close to one another. A generalization of the LPB descriptor in the form of a local ternary pattern (LTP) was proposed as one simple way of partially alleviating this problem [35]. In particular, instead of mapping neighbourhood pixels to binary digits, to produce a LTP the mapping is done to a ternary digit i.e. without loss of generality, to 0 , 1 , or 2 . A pixel is mapped onto 0 or 2 respectively if its value is smaller or greater than that of the central pixel by at least a certain amount (this threshold is a free parameter), and to 1 otherwise. The latter, additional value can be seen as representing neighbourhood pixels sufficiently similar to 
the central one. The remainder of the method, that is the aggregation of local descriptors into histograms over blocks, and the concatenation of these to form a holistic representation, is performed just as in the original LBP based method.

Numerous other derivative representations have been proposed for use in different domains, including over-complete LBPs (OCLBP) [36], volume LBPs (VLBP) [37] and other $3 \mathrm{D}$ variants [38], and RGB-LBPs [39].

\section{TECHNICAL CONTRIBUTIONS}

In this section we describe the two key technical contributions of the present work.

\section{A. Adaptive weighting}

From the earliest days of research on face recognition (both human and machine based) it has been appreciated that different regions of the face, and thus its image representation, do not contain the same amount of usual, discriminative information content. Speaking on a semantic level, we can say that regions around salient facial features are particularly important [40]. Indeed, this is what leads to us calling them salient. While some previous work has sought to exploit this explicitly, by detecting such semantically meaningful elements (usually the eyes, the mouth, and the nose), a limitation of these approaches is that a bottleneck is inherently introduced: facial feature detection itself becomes an extremely hard problem in unconstrained and severe imaging conditions. An alternative is to move away from semantics and focus on low-level proxy indicators of saliency, namely high frequency content [41], which does not exhibit the same potential bottleneck. This is the approach adopted in the present work.

Yet, the original LBP descriptor does not account for the aforementioned observation, either implicitly or explicitly, with descriptors associated with all image loci contributing with equal weight to the overall representation of appearance. Consequently, the overwhelming proportion of the face which exhibits uniform appearance and thus contains little discriminative information, disproportionately smooths data by pulling the representation towards the average. To address this limitation we introduce the first modification to the original algorithm by weighting the contribution of each LBP pattern by a monotonically increasing function of the average gradient of its neighbourhood. In particular, we propose and henceforth employ the following weighting function:

$$
w_{g}(\bar{G})=e^{n \bar{G}}-1,
$$

where $w_{g}$ is the weight associated with a particular image locus, $\bar{G}$ is the average gradient magnitude in the square neighbourhood of the locus, and $n$ a parameter learnt from a training corpus. This functional form is motivated by the common exponential response of many physiological perceptual systems, normalized by subtraction to ensure that uninformative, entirely uniform regions make no contribution. Of course the final histogram is normalized not through the division by a total loci count but by the sum of the corresponding loci weights.

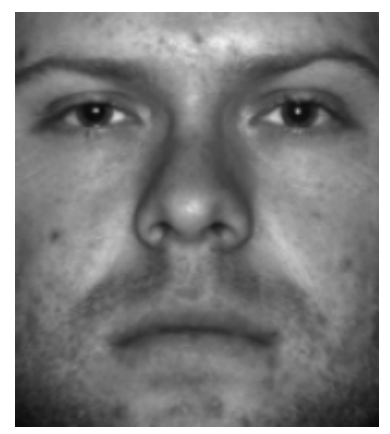

(a) Example face

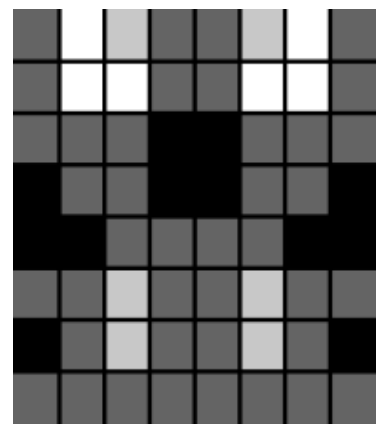

(b) Weighting matrix
Fig. 2. The first improvement on the original LBP based method proposed in this paper exploits the heterogeneity of discriminative information across a face. In particular, instead of having each image locus contribute to the overall appearance representation equally, we weight loci (i.e. the corresponding LBPs) according to the local neighbourhood gradient magnitude, associated with greater discriminative content.

\section{B. Topological uncertainty}

The second limitation of LBP based face recognition algorithms we highlight in this work concerns spatial discretization. Recall that the original method divides an input image into regions, each of which is described by a histogram of the corresponding area's LBPs which are the concatenated to describe the image as a whole. One of the weaknesses of this approach lies in the partial loss of spatial information across the image. Indeed, this has been recognized by other researchers in the past [33]. An approach proposed to addressed in the form of overlapping regions has shown itself successful - indeed the same idea has been been exploited in a variety of domains when there is either spatial or temporal relatedness between different signal segments, including object recognition [42], face recognition [43], and document analysis [44].

Nevertheless, merely overlapping neighbouring regions does not address the problem associated with the 'hard' assignment of pixel membership to different regions - each pixel is deemed either to be within the region or outside of it, regardless of its precise position. In other words, a pixel within a region is treated exactly the same, in terms of its contribution to the the description of the region, if it is near the region boundary or close to the centre of the region. This means that the representation is highly sensitive to registration imprecisions whereby a small change in input can effect a large change in the corresponding output (representation); yet, precise registration of faces in challenging condition is notoriously difficult to ensure [45].

Although the nature of the phenomenon of interest here is different, there is a conceptual parallel with the problem considered in the previous section, which is reflected in the approach we propose. In particular, we apply an additional layer of local descriptor weighting but this time round based on the stability of the membership its locus to a particular 
region. The weighting is thus of the form:

$$
w_{b}(d)=e^{-(d / \sigma)^{2}},
$$

where $d$ is the distance of the locus from the centre of a region to which histogram it contributes with the weight $w_{b}$, and $\sigma$ a free parameter, as before learnt from a training corpus. Effectively the membership now becomes fuzzy rather than hard binary [46].

\section{EMPIRICAL EVALUATION}

In this section we describe the experiments we conduced to evaluate and assess the performance of the proposed ideas, summarize the most important findings, and discuss their implications for practical application and future research. We being by introducing the data sets we adopted for the evaluation.

\section{A. Data}

We evaluated the proposed representation on the YaleB database [47], [48]. This is a challenging data set used as a standard benchmark for the comparison of face recognition algorithms in terms of their robustness to severe illumination changes [43], [49]. The variation in the direction of the dominant light source illuminating a face is extreme: its azimuth varies from $-130^{\circ}$ to $130^{\circ}$, and its elevation from $-40^{\circ}$ (i.e. pointing upwards) to $90^{\circ}$ (i.e. directly overhead, pointing downwards), giving a total of 64 different illumination conditions. Notice that the face is sometimes illuminated from the rear lateral direction (and thus hardly illuminated at all), that extreme cast shadows are often present as are highly bright saturated image regions. Some of these challenges have already been illustrated in Figure 3. The database does not include any intentional variation in facial expression, but some variation exists nonetheless, mainly in the form of squinting when the subject is facing the dominant light source.

\section{B. Results}

We adopt the standard protocol whereby data is divided into training, test, and validation (sub)sets. All parameter learning is performed using training data and optimized over on the test set, with validation data used only for the final performance assessment. It is important to emphasise that in order to ensure that no overfitting takes place training, test, and validation sets are non-overlapping. In addition to no image being included in two of these, we also do not even use the same person (i.e. not even different images of the person) in multiple sets, lest there be any implicit overfitting effected by means of learning some characteristic preserved by the used image representations.

We begin by learning the size of regions into which an input image is divided for the purpose of LBP histogram building. The plot in Figure 4 shows the effect of varying this parameter. As expected, a coarse division into a small number of large regions produces inferior results. Increasing the number of regions, i.e. reducing the size of local blocks,

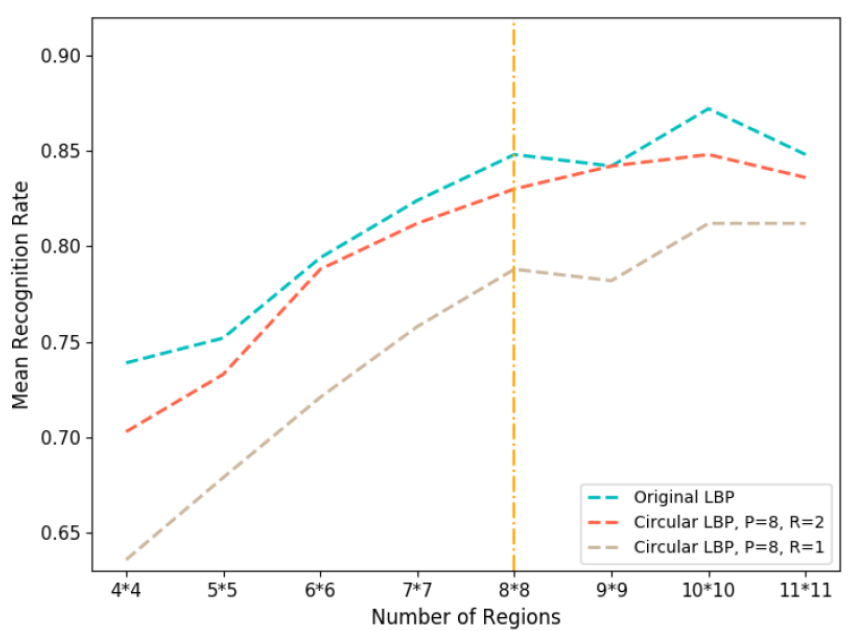

Fig. 4. Optimization of the number of regions images are divided into for LBP histogram construction.

improves performance which tails off for a greater number than $8 \times 8$ blocks. This is consistent with the previous reports in the literature and thus henceforth the block size is fixed to this value.

Similarly, the free parameters of our two weighting schemes, namely the gradient based weighting introduced in Section III-A (n.b. for computational reasons gradient estimation was performed using the Sobel operator) and the fuzzy region membership described in Section III-B, are also learnt from training and test data set. Based on the results shown the following values are used henceforth: gradient scaling $n=120$, linear region overlap proportion $\rho_{o}=0.3$, and Gaussian fuzzy membership drop-off rate $\sigma=0.4$.

Finally, we turn our attention to the evaluation of trained algorithms on the validation data. To facilitate a more nuanced analysis we followed previous work in considering separately six different subsets of the data, formed according to severity of illumination conditions present [50]. The least challenging

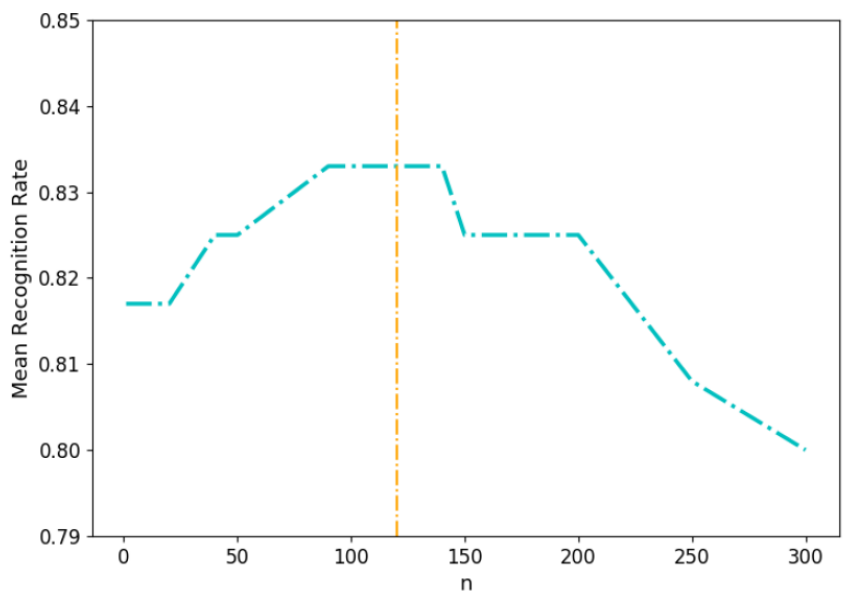

Fig. 5. Optimization of the value of the free parameter in our gradient based local descriptor weighting. 

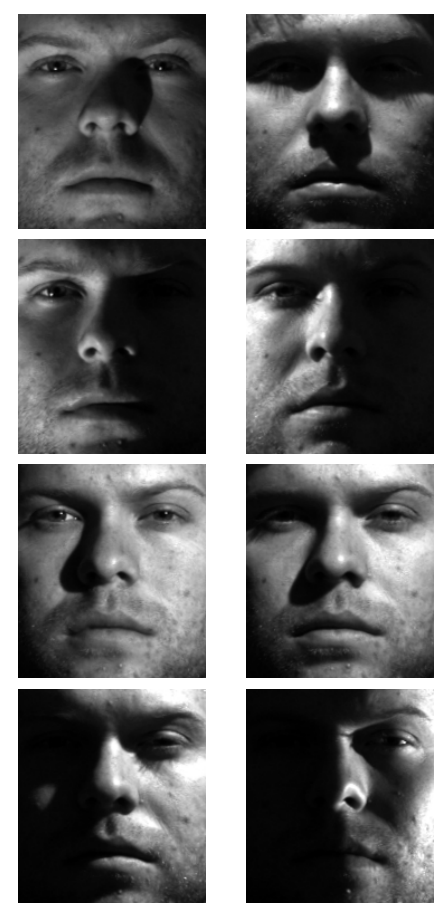
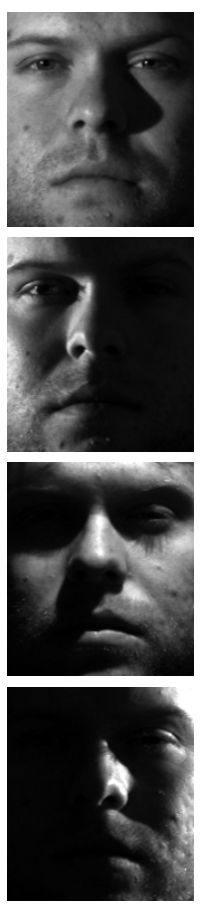
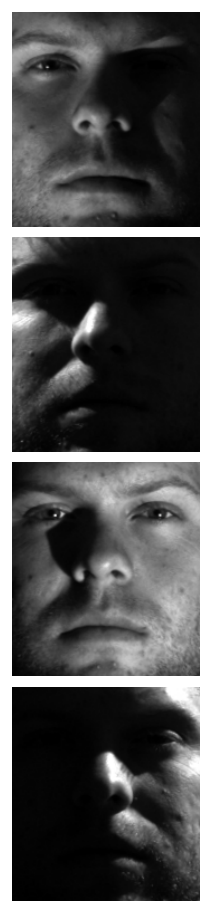
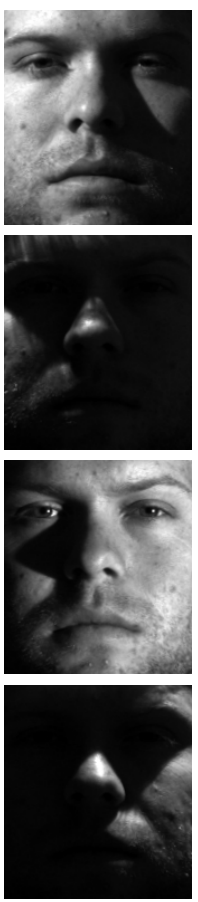
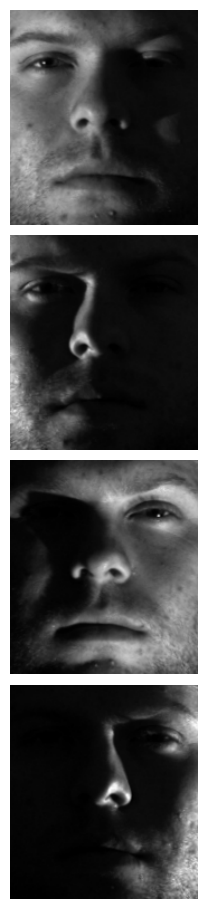
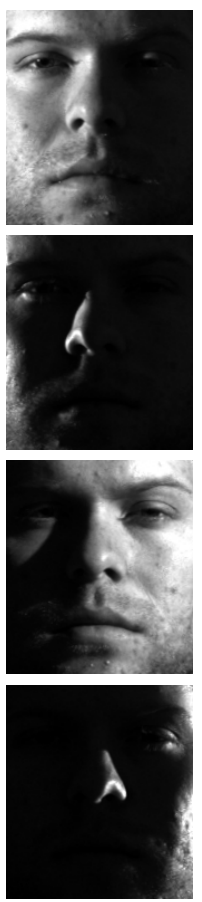
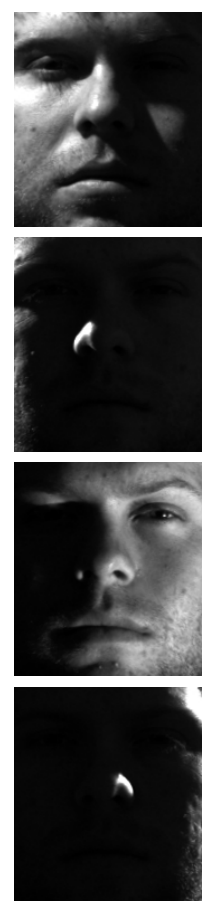

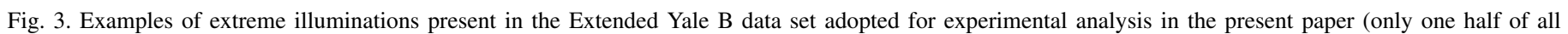
conditions are shown due to space constraints).

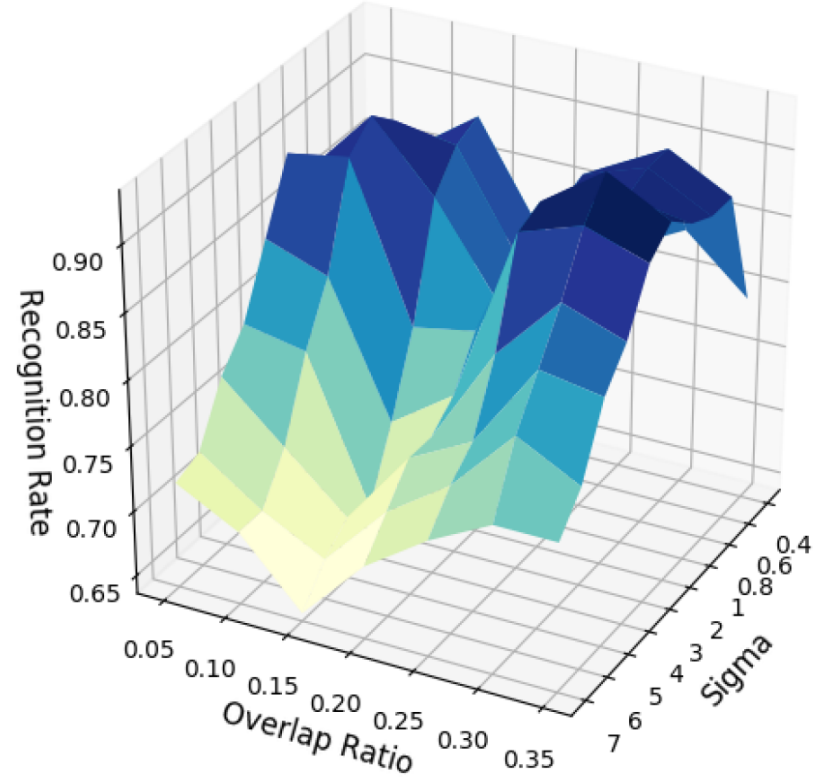

Fig. 6. Optimization over the two interrelated parameters in our Gaussian weighting of LBP contributions based weighting of LBP contributions to different regions' histograms - the width of the Gaussian function and the overlap between neighbouring regions.

two subsets were used for training, and the remaining four for validation. The main results are summarized in Table I. Firstly, observe that already for the least challenging of the four subsets (Subset 3) the proposed approach decreases the average error rate from $15 \%$ to $2 \%$, i.e. for nearly an order of
TABLE I. SUMMARY OF EXPERIMENTAL COMPARISON OF THE PROPOSED METHOD WITH THE ORIGINAL LBP BASED ALGORITHM.

\begin{tabular}{l|cccc}
\hline & \multicolumn{4}{|c}{ Validation subsets by difficulty } \\
& Subset 3 & Subset 4 & Subset 5 & Subset 6 \\
\hline Original & 0.85 & 0.70 & 0.24 & 0.07 \\
Proposed & 0.98 & 0.96 & 0.67 & 0.30 \\
\hline
\end{tabular}

magnitude. The benefits of the proposed modification of the overall framework becomes even more apparent as the severity of challenge increases. Already for Subset 5 the original method's performance is extremely poor and effectively useless for any practical application, with our method exhibiting a far more graceful drop-off (nearly though not quite on par with the original method's performance on the least challenging subset).

We also examined the behaviour of the proposed modifications in the presence of registration errors by synthetically introducing them (validation Subset 3 was used in this experiment). The corresponding results are illustrated by the surface plot in Figure 7. As expected from theory and the premises of the proposed ideas, an impressive degree of robustness is demonstrated. Even a significant shift of 6 pixels both in the horizontal and vertical directions effects a reduction of only approximately $11 \%$ in the average rank-1 recognition rate, resulting in performance comparable with the original method and unrealistic, perfect registration. 


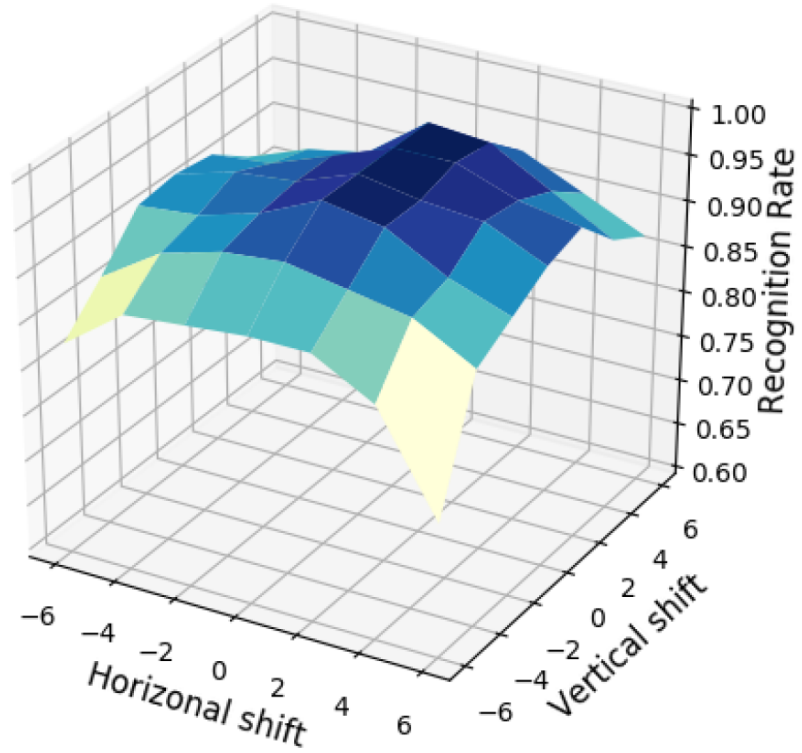

Fig. 7. Robustness of the proposed method to registration errors i.e. image misalignment. Good resilience is witnessed by graceful performance degradation and the maintenance of high recognition rate even for large errors.

\section{SUMMARY AND CONCLUSIONS}

. The successes of the local binary descriptor across a range of different applications make it a popular choice in many image recognition tasks, not the least significant of which is face recognition. Though much work has been conducted on devising improvements based on the original idea, all of the extensions described in the existing literature inherit some of the fundamental limitations. In this work we discussed these and proposed novel ways of addressing them in a principled fashion. Specifically, we introduce (i) gradient based weighting of local descriptor contributions to region based histograms as a means of avoiding data smoothing by non-discriminative image loci, and (ii) Gaussian fuzzy region membership as a means of achieving robustness to registration errors. Importantly, the nature of these contributions allows the proposed techniques to be combined with the existing extensions to the LBP descriptor thus making them universally recommendable. Effectiveness is demonstrated on the notoriously challenging Extended Yale B face corpus, with the benefits being most prominent when they are needed the most: in the most challenging conditions characterized by severe illumination effects and significant registration errors.

\section{REFERENCES}

[1] W. Zhao, R. Chellappa, P. J. Phillips, and A. Rosenfeld, "Face recognition: A literature survey." ACM Computing Surveys, vol. 35, no. 4, pp. 399-458, 2004

[2] Y. Gao, J. Ma, and A. L. Yuille, "Semi-supervised sparse representation based classification for face recognition with insufficient labeled samples." IEEE Transactions on Image Processing, vol. 26, no. 5, pp. 2545-2560, 2017.

[3] J. Fan and O. Arandjelović, "Employing domain specific discriminative information to address inherent limitations of the LBP descriptor in face recognition." In Proc. IEEE International Joint Conference on Neural Networks, 2018.

[4] Z. Dong, C. Jing, M. Pei, and Y. Jia, "Deep CNN based binary hash video representations for face retrieval." Pattern Recognition, 2018.

[5] T. Kanade, "Picture processing system by computer complex and recognition of human faces." Ph.D. dissertation, Kyoto University, 1973.

[6] M. Turk and A. Pentland, "Eigenfaces for recognition." Journal of Cognitive Neuroscience, vol. 3, no. 1, pp. 71-86, 1991.

[7] R. Chellappa, C. L. Wilson, and S. Sirohey, "Human and machine recognition of faces: A survey." Proceedings of the IEEE, vol. 83, no. 5, pp. 705-740, 1995.

[8] T. Fromherz, P. Stucki, and M. Bichsel, "A survey of face recognition." MML Technical Report., no. 97.01, 1997.

[9] G. J. Edwards, T. F. Cootes, and C. J. Taylor, "Face recognition using active appearance models." In Proc. European Conference on Computer Vision, vol. 2, pp. 581-595, 1998.

[10] S. Edelman and A. J. O'Toole, "Viewpoint generalization in face recognition: The role of category-specific processes." Computational, geometric, and process perspectives on Facial cognition: Contexts and Challenges, 1999.

[11] The Register, "Face recognition useless for crowd surveillance." The Register, 27 September, September 2001.

[12] A. S. Tolba, A. H. El-Baz, and A. A. El-Harby, "Face recognition: A literature review." International Journal of Signal Processing, vol. 2, no. 2, pp. 88-103, 2006.

[13] O. Arandjelović, "A framework for improving the performance of verification algorithms with a low false positive rate requirement and limited training data." In Proc. IEEE/IAPR International Joint Conference on Biometrics, 2014, DOI: 10.1109/BTAS.2014.6996275.

[14] P. Sinha, B. Balas, Y. Ostrovsky, and R. Russell, "Face recognition by humans: Nineteen results all computer vision researchers should know about." Proceedings of the IEEE, vol. 94, no. 11, pp. 1948-1962, 2006.

[15] J. B. Wilmer, L. Germine, C. F. Chabris, G. Chatterjee, M. Williams, E. Loken, K. Nakayama, and B. Duchaine, "Human face recognition ability is specific and highly heritable." Proceedings of the National Academy of Sciences, vol. 107, no. 11, pp. 5238-5241, 2010.

[16] R. He, T. Tan, L. Davis, and Z. Sun, "Learning structured ordinal measures for video based face recognition." Pattern Recognition, vol. 75, pp. 4-14, 2018.

[17] J. Tang, Z. Li, and X. Zhu, "Supervised deep hashing for scalable face image retrieval." Pattern Recognition, vol. 75, pp. 25-32, 2018.

[18] O. Arandjelović, "Colour invariants under a non-linear photometric camera model and their application to face recognition from video." Pattern Recognition, vol. 45, no. 7, pp. 2499-2509, 2012.

[19] K.-K. Huang, D.-Q. Dai, C.-X. Ren, Y.-F. Yu, and Z.-R. Lai, "Fusing landmark-based features at kernel level for face recognition." Pattern Recognition, vol. 63, pp. 406-415, 2017.

[20] T. Pei, L. Zhang, B. Wang, F. Li, and Z. Zhang, "Decision pyramid classifier for face recognition under complex variations using single sample per person." Pattern Recognition, vol. 64, pp. 305-313, 2017.

[21] Y.-F. Yu, D.-Q. Dai, C.-X. Ren, and K.-K. Huang, "Discriminative multi-scale sparse coding for single-sample face recognition with occlusion.” Pattern Recognition, vol. 66, pp. 302-312, 2017.

[22] X. Yu, Y. Gao, and J. Zhou, "Sparse 3D directional vertices vs continuous 3D curves: efficient 3D surface matching and its application for single model face recognition." Pattern Recognition, vol. 65, pp. 296-306, 2017.

[23] G. Gao, J. Yang, X.-Y. Jing, F. Shen, W. Yang, and D. Yue, "Learning robust and discriminative low-rank representations for face recognition with occlusion." Pattern Recognition, vol. 66, pp. 129-143, 2017.

[24] X. Wu, Q. Li, L. Xu, K. Chen, and L. Yao, "Multi-feature kernel discriminant dictionary learning for face recognition." Pattern Recognition, vol. 66, pp. 404-411, 2017. 
[25] Z. Fan, D. Zhang, X. Wang, Q. Zhu, and Y. Wang, "Virtual dictionary based kernel sparse representation for face recognition." Pattern Recognition, vol. 76, pp. 1-13, 2018.

[26] I. Schlag and O. Arandjelović, "Ancient Roman coin recognition in the wild using deep learning based recognition of artistically depicted face profiles." In Proc. IEEE International Conference on Computer Vision, pp. 2898-2906, 2017.

[27] A. T. Lopes, E. de Aguiar, A. F. de Souza, and T. Oliveira-Santos, "Facial expression recognition with convolutional neural networks: coping with few data and the training sample order." Pattern Recognition, vol. 61, pp. 610-628, 2017.

[28] Y. Li, G. Wang, L. Nie, Q. Wang, and W. Tan, "Distance metric optimization driven convolutional neural network for age invariant face recognition." Pattern Recognition, vol. 75, pp. 51-62, 2018.

[29] O. Arandjelović, "Learnt quasi-transitive similarity for retrieval from large collections of faces." In Proc. IEEE Conference on Computer Vision and Pattern Recognition, pp. 4883-4892, 2016.

[30] D. G. Lowe, "Distinctive image features from scale-invariant keypoints." International Journal of Computer Vision, vol. 60, no. 2, pp. 91-110, 2003.

[31] M. Heikkilä, M. Pietikäinen, and C. Schmid, "Description of interest regions with local binary patterns." Pattern Recognition, vol. 42, no. 3, pp. 425-436, 2009.

[32] R. S. Ghiass, O. Arandjelović, A. Bendada, and X. Maldague, "Infrared face recognition: a literature review." In Proc. IEEE International Joint Conference on Neural Networks, pp. 2791-2800, 2013.

[33] T. Bouwmans, C. Silva, C. Marghes, M. S. Zitouni, H. Bhaskar, and C. Frelicot, "On the role and the importance of features for background modeling and foreground detection." arXiv, p. 1611.09099, 2016.

[34] X. Tan and B. Triggs, "Enhanced local texture feature sets for face recognition under difficult lighting conditions." In Proc. International Workshop on Analysis and Modeling of Faces and Gestures, pp. 168182, 2007.

[35] J. Trefnỳ and J. Matas, "Extended set of local binary patterns for rapid object detection." In Proc. Computer Vision Winter Workshop, pp. 1-7, 2010.

[36] O. Barkan, J. Weill, L. Wolf, and H. Aronowitz, "Fast high dimensional vector multiplication face recognition." In Proc. IEEE International Conference on Computer Vision, pp. 1960-1967, 2013.

[37] G. Zhao and M. Pietikainen, "Dynamic texture recognition using local binary patterns with an application to facial expressions." IEEE Transactions on Pattern Analysis and Machine Intelligence, vol. 29, no. 6, pp. 915-928, 2007.
[38] H. Tang, B. Yin, Y. Sun, and Y. Hu, "3D face recognition using local binary patterns.” Signal Processing, vol. 93, no. 8, pp. 2190-2198, 2013.

[39] H. H. Vo and A. Verma, "Discriminant color texture descriptors for diabetic retinopathy recognition." In Proc. IEEE International Conference on Computational Photography, pp. 309-315, 2016.

[40] O. Arandjelović and R. Cipolla, "A new look at filtering techniques for illumination invariance in automatic face recognition." In Proc. IEEE International Conference on Automatic Face and Gesture Recognition, pp. 449-454, 2006.

[41] O. Arandjelović, "Making the most of the self-quotient image in face recognition." In Proc. IEEE International Conference on Automatic Face and Gesture Recognition, 2013, DOI: 10.1109/FG.2013.6553708.

[42] W. Rieutort-Louis and O. Arandjelović, "Bo(V)W models for object recognition from video." In Proc. International Conference on Systems, Signals and Image Processing, pp. 89-92, 2015.

[43] O. Arandjelović, "Unfolding a face: from singular to manifold." In Proc. Asian Conference on Computer Vision, vol. 3, pp. 203-213, 2009.

[44] A. Beykikhoshk, O. Arandjelović, D. Phung, and S. Venkatesh, "Discovering topic structures of a temporally evolving document corpus." Knowledge and Information Systems, vol. 55, no. 3, pp. 599-632, 2018.

[45] O. Arandjelović and R. Cipolla, "Face set classification using maximally probable mutual modes." In Proc. IAPR International Conference on Pattern Recognition, pp. 511-514, 2006.

[46] O. R. Bidder, O. Arandjelović, F. Almutairi, E. Shepard, S. Lambertucci, and R. P. Wilson, "A risky business or a safe BET? A fuzzy set event tree for estimating hazard in biotelemetry studies." Animal Behaviour, vol. 93, no. 7, pp. 143-150, 2014.

[47] A. S. Georghiades, P. N. Belhumeur, and D. J. Kriegman, "From few to many: illumination cone models for face recognition under variable lighting and pose," IEEE Transactions on Pattern Analysis and Machine Intelligence, vol. 23, no. 6, pp. 643-660, 2001.

[48] D. Pham, O. Arandjelović, and S. Venkatesh, "Achieving stable subspace clustering by post-processing generic clustering results." In Proc. IEEE International Joint Conference on Neural Networks, pp. 23902396, 2016.

[49] Y. Biadglgne and O. Arandjelović, "Face filtering - insights from realworld data." In Proc. International Conference on Systems, Signals and Image Processing, pp. 65-68, 2015.

[50] O. Arandjelović, "Gradient edge map features for frontal face recognition under extreme illumination changes." In Proc. British Machine Vision Conference, 2012, DOI: 10.5244/C.26.12. 